\title{
METHODOLOGICAL ANALYSIS OF AUTOMATION AND ROBOTIZATION FOR THE PROCESS OF PLACING AND CONSOLIDATION OF A CONCRETE MIXTURE
}

\author{
Prof. Dr.-Ing./Univ. Tokio Thomas Bock, Dipl.-Ing. Serguei Voltchkov \\ Technical University Munich \\ Chair for Building Realization and Informatics \\ Arcisstrasse 21, D-80333 Munich, Germany \\ Fon +498928922100 Fax +498928922102 \\ e-mail:svoltchkov@bri.arch.tu-muenchen.de
}

\begin{abstract}
Transportation, distribution and consolidation of a concrete mixture demands use of technical resources, able to supply and to place a concrete mixture into large structures, erected at determined height under zero level, allowing the concrete to keep its required properties. Using of robots in construction promotes a heightening of work's productivity, improvement of quality of operational execution and reduction of danger for workers. The application of manipulators in monolithic construction allows to eliminate hand-operated work and to automize processes of concrete mixture's stacking.
\end{abstract}

Keywords: automation, robot, construction, conctere, placing, consolidation.

\section{TECHNOLOGICAL PREMISES FOR AUTOMATION AND ROBOTIZATION OF PLACING PROCESS OF A CONCRETE MIXTURE}

The technological process of buildings' construction from monolithic concrete and ferro-concrete consists of interdependent procuring and building processes. To procuring processes, which are carried on in industrial conditions, one can mention manufacture of shuttering elements, of reinforcing cages and preparation of concrete mixture. Building processes include shuttering assembly from separate elements, mounting of an armature, transportation of concrete mixture, placing and consolidation of concrete mixture, drift of concrete, disassembly of shuttering. Transporting, feeding and distribution of concrete mixture at a level of a shuttering working area demands means, able to provide and to stack mixture below zero mark in constructions, raised at defined height, with the large sizes on square, volume and permitting thus to save its required properties. In this case processes of transporting, feeding and distribution should be continuous and completely meet the given rate of concreting.

In practice of monolithic construction during completing buildings with the purpose to lower expenditures for heavy hand-operated transactions during feeding and distribution of concrete mixture one use the following means:

- cranes and elevators;

- devices for gravitational feeding and distribution of concrete mixture;

- pipelines and concrete placers.

The experience displays, that the wide application of pipeline transportational technique have to be full coordinated with basic directions of intensification and complex mechanization of concrete operations, allowing to reduce the cost price of stacking at simultaneous magnification of development accounting to one worker more than twice, to lower complexity of monolithic buildings' construction, to increase efficiency of a transactions, to remove losses of concrete mixture at overloads up to $5 \%$, to improve conditions and labor safety, to increase quality and culture of manufacture [1].

The importance of this direction is determined by the point that now there is an especially sharp problem of rational using of a manpower in connection with the lack of a possibility of manufacturing expansion at the expense of increasing number working.

Thus, using of robots in construction promotes a heightening of work's productivity, improvement of quality of operational execution and reduction of danger for workers.

\section{ANALYSIS OF EXISTING TOOLS OF AUTOMATION AND ROBOTIZATION FOR THE PROCESS OF PLACING AND CONSOLIDATION OF A CONCRETE MIXTURE}

The new opportunities of using concrete pumps were discovered with apparition of the whole family of 
machines equipped with a manipulator of the concrete pipeline. The development of the idea of a mechanical manipulation of the concrete pipeline has resulted in the creation of manipulators mounted on a final part of a stationary concrete pipeline, at a significant distance from the concrete pump. Such manipulators can be carried on supporting constructions such as tower-cranes, portable hydraulic cranes etc. [2].

Wide application takes place in truck mixers, equipped with distributive booms (Figure 1). The leading manufacturers of such equipment are the firms of Germany [3 - 5]. The use of distributive cantilever arms in a combination with well developed logistics on the building spot results in an essential save of time; that is one of the basic factors, both in monolithic construction, and in economy in general.

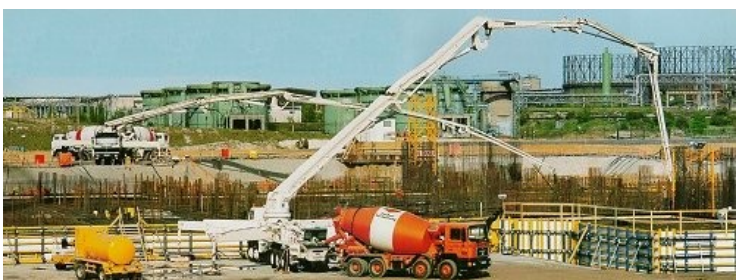

Figure 1. Distributive booms on the building site [6]

The significant effect will be brought about by devices based on microprocessors in manipulation systems to place concrete mixtures and in assembly towers with telescopic or articulated cantilever arms on a special pneumowheel chassis [7, 8]. The computer provides reliable performance of the program of control of hydraulics and both observance of necessary height and speed of moving of the end of a mast. The radio control of the process is possible only in that case, when on a building spot there are no signals or noises able to disturb radio communication $[9,10]$.

In Japan has been developed a robot for horizontal and vertical distribution of a concrete mixture in shuttering [11]. The robot is fixed with the help of the holder on a temporary or constant platform, which may have any form and size. For transportation of the concrete there exists pipes with demountable connections, fixed on the arms of the manipulator. On the last joint there is a flexible sleeve, which is connected to the concrete pipeline. The panel of control is made in a mobile form and is located on the fourth element. The remote control of the robot can also be used. The manipulator has vibrators to compress the concrete mixture or devices for finishing works.

The building robots are able to function in various technological processes in certain conditions, more often under strict programs. However many tasks require the presence of elements of artificial intelligence in the robot. In such systems the mechanical executive elements, controlled by "intellectual" computers with advanced subsystems of recognition of visual, tactile or other information, cooperate with the external environment, which is sometimes complex and uncertain. In this case, mathematical modeling of operations of the robot on the basis of the information describing a building spot and the structure of the robot self is the best solution to the decision of a task concerning the synthesis of a control system. The model has a function of information interpretation, and the computer decides the election of its physical, geometrical, symbolical and logical representations for estimation of building operations, for planning of optimal trajectories of movement and control of task's performance [12].

\section{REQUIREMENTS TO ROBOT SYSTEM FOR STACKING OF CONCRETE MIXTURE IN A SHUTTERING}

As a conclusion from above-stated exists a necessity for development of a robot system for transportation, placing and consolidation of concrete mixture. It is necessery to formulate the requirements, which a robot system for the indicated purposes should fullfill:

- possibility of the definition of a concreting building's configuration (its overall dimensions, disposition of an armature etc.) before stacking of concrete mixture.

- the kinematic configuration of manipulators of the robot system should ensure appropriate performance of a given configuration.

- $\quad$ stability of manipulators in case of influence of fixed and variable wind loads, especially at highaltitude construction.

Robot system should have a control system, which

- ensures the stability of given trajectory of manipulator's motion in view of exposures;

- regulates a speed of manipulator's motion according to shuttering rise;

- regulates pressure and speed of concrete mixture's feeding in concrete pipeline depending on structure of mixture and climatic conditions;

- regulates frequency of concrete mixture's vibrating and depth of the vibrator's immersing depending on structure of concrete mixture and climatic conditions.

Robot system should have an informational system, which

- identifies obstacles for moving of manipulators;

- determines such parameters of concrete mixture as density, temperature, pressure and feeding speed through the pipeline and other;

- determines such parameters of an environment as temperature, damp, wind loads and other;

- determines frequency of vibrating of the manipulator and depth of its immersing in concrete mixture.

The possibility of transferring in case of emergency into hand-operated control from the remote control is also necessary.

The functional diagram of robot system is 
demonstrated on Figure 2.

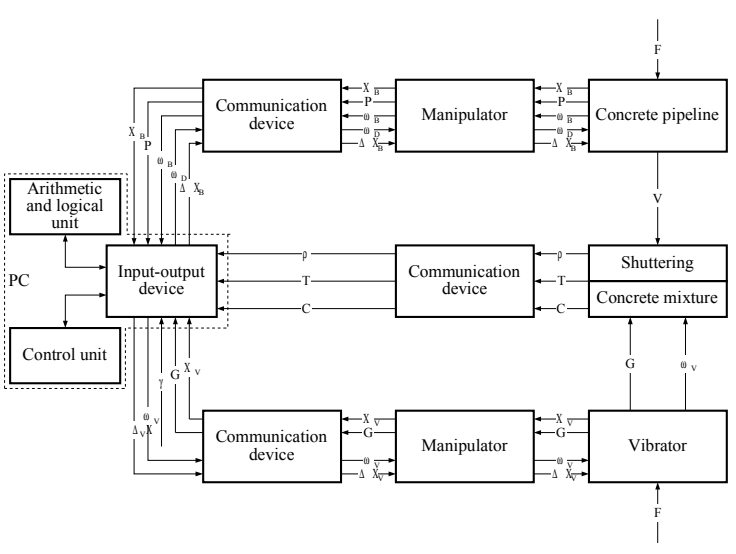

Figure 2. Functional diagram of robot system

\section{COMPLEMENTARY DOMAINS OF ROBOT SYSTEM'S APPLICATION ON THE BASIS OF MANIPULATORS FOR PLACING OF CONCRETE MIXTURE}

Manipulators, equipped with the additional working instrument, will be recently used also at suppression of fires or as superflexible cranes for transportation of heavy weights in complicated conditions. The mounting of a cradle on a gripper of the manipulator allows to use the manipulator as a flexible working platform, and it is possible to carry out the inspections of the lower parts of automobile and railway bridges, cleaning of facades or windows of high-altitude houses and other service operations. And other example of using of the large manipulators in the field of service is designed by Putzmeister corporation (Germany) robot for washing of planes SKYWASH (Figure 3), with its help the significant reducing of wash time is achieved and accordingly and non-working of planes, that is accompanied by reducing of aircorporations' expenditures.

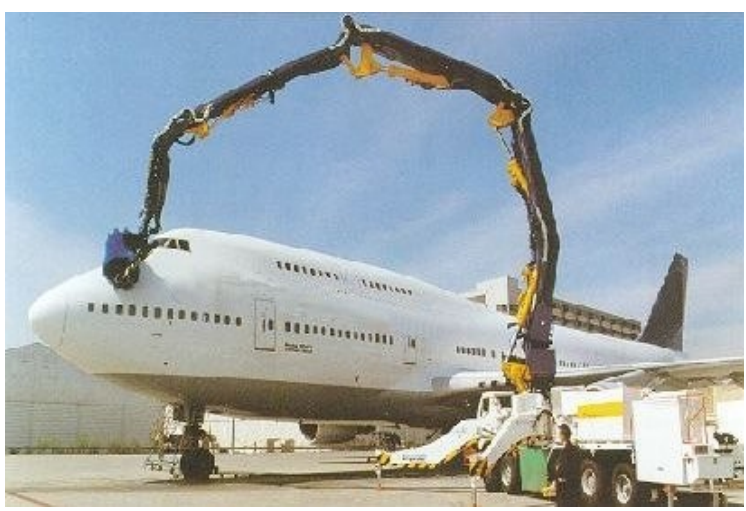

Figure 3. SKYWASH-robot at work [13]

Besides the authors offer to use the following variants of robot systems on the basis of the large manipulators in different industries.

Pipeline transportation and power engineering:

- diagnostics of gas and oil pipelines and their renovation in case of flaws;
- clearing pipes of power plants, their diagnostics and renovation;

- research of dams in case of flaws and their renovation.

Construction industry:

- welding and other aspects of installation works at a large height and in conditions of insufficiency of working space;

- construction of tunnels;

- renovation operation;

- diagnostics of buildings' facades and their sanation.

Transport:

- loading and unloading of the ships and trains in conditions of insufficiency of working space;

- clearing underwater parts of the ships.

Antropogeneous catastrophes:

- research of area in order to scrutinize its infection;

- clearing of the rivers', lakes' and seas' surfaces from oil pollution.

\section{REFERENCES}

[1] Pishtchalenko J.A. Technique of buildings' construction. - Kiev: Wishtcha schkola, 1982. - 192 p.

[2] Hajutin J.G. Monolithic concrete: the "knowhow" of operations.- Moscow: Strojizdat, 1991. $576 \mathrm{p}$.

[3] The request for the invention. Germany 4306127, B 25 J 005/00. Großmanipulator, insbesondere für Autobetonpumpen. 01.09.94.

[4] The request for the invention. Germany 4412643, B 25 J 005/00. Großmanipulator, insbesondere für Autobetonpumpen, sowie Verfahren zu dessen Handhabung. 02.03.95.

[5] The request for the invention. Germany 4412635, H 04 Q 009/00. Fernsteueranordnung, insbesondere für Förder-, Spritz- und Verteilermaschinen für Beton und Mörtel. 19.10.95.

[6] http://www.putzmeister.de/d/produkte/bpauto/def ault.asp, access: June 2000.

[7] ACM-System PM, MT1067, Datenblatt der Fa. Putzmeister.

[8] Geschäftsbereich Daimler-Benz, Unternehmensbereich AEG. - 1988. - S. 36.

[9] Prototype. Germany 29505549, F 21 V 033/00. Fernsteuergerät für mobile Arbeitsmaschinen. 20.07.95.

[10]Neue Prop-Funkfernsteuerung // Baumarkt, 1998, № 7, S. 20.

[11] Finch M.C. Concrete Pumping a Growth Industry // Civil Engineering. Great Britain, 1985, № 6, P. 16-19

[12] Microprocessors in systems of building engineering automation. Bulgakow A.G., Gerner I. Kaden R. - M.: VNIINTPI, 1991. - 52 p. [13] http://www.putzmeister.de/d/produkte/rh/default. asp, access: June 2000. 